\title{
Designing and validating a measuring tool for the factors affecting the distribution of hospitals' intensive care beds in Iran
}

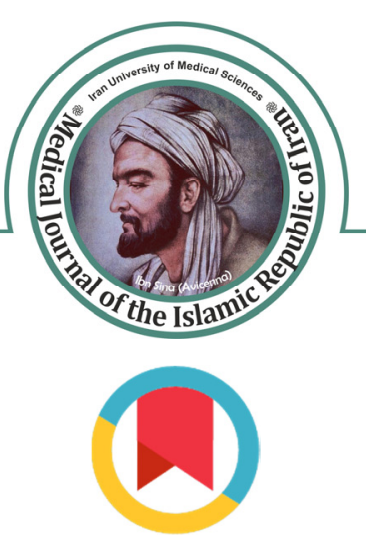

\begin{abstract}
Nahid Farrokhyar ${ }^{1}$, Khalil Alimohammadzadeh*1,2 ${ }^{\text {Ali Maher }}{ }^{3}$, Seyed Mojtaba Hosseini ${ }^{1}$, Mohammadkarim Bahadori ${ }^{4}$
\end{abstract}
Received: 28 Apr 2019

Published: 5 Aug 2019

\section{Abstract}

Background: Hospital services are the most expensive medical service in modern health care systems. Intense care beds, in particular, are more important. The present study was conducted to design and validate a measuring tool for the factors affecting the distribution of hospitals' intensive care beds in Iran.

Methods: In this mixed method study, first, all known factors affecting the distribution of hospitals' intensive care beds were extracted by reviewing related literature. Then, all 60 confirmed items were categorized into different dimensions. Face validity and content validity of the questionnaire was done by 20 medical experts through qualitative and quantitative methods. Validity and reliability indices, content validity index (CVI), content validity ratio (CVR), and Cronbach's alpha were measured. SPSS software were used for data analysis and significance level was set at less than .05 .

Results: From the 60 suggested items, 34 were confirmed by the expert panels and all items had CVR and CVI scores higher than 0.83 and 0.81 , respectively. CVR and CVI for all 34 items were 0.88 and 0.89 , respectively. Also, Cronbach's alpha coefficient (0.75) indicated a suitable internal consistency. The value of S-CVI / Ave was also calculated to be 0.92 .

Conclusion: In this study, a valid tool was designed to identify the factors affecting the distribution of hospitals' intensive care beds. This tool consists of 6 dimensions: demographic, geographic, economic, sociopolitical, organizational, and constructional.

Keywords: Intensive care beds, Rationing intensive care beds, Distribution of beds

Conflicts of Interest: None declared

Funding: None

*This work has been published under CC BY-NC-SA 1.0 license.

Copyright $\odot$ Iran University of Medical Sciences

Cite this article as: Farrokhyar N, Alimohammadzadeh Kh, Maher A, Hosseini SM, Bahadori M. Designing and validating a measuring tool for the factors affecting the distribution of hospitals' intensive care beds in Iran. Med J Islam Repub Iran. 2019 (5 Aug);33:79. https://doi.org/10.47176/mjiri.33.79

\section{Introduction}

A major part of the budget of ministries of health in all countries will be allocated to hospitals (1). Hospital services are the most expensive medical service in modern health care systems (2).

However, inappropriate allocation of these expensive resources across the globe has led to resource consumption (3). In this regard, the distribution of hospital beds can be used as one of the indicators of the distribution of services (4). Intensive care beds are also highly important. The need for intensive care units around the world is in-

\footnotetext{
Corresponding author: Dr Khalil Alimohammadzadeh,dr_khalilamz@yahoo.com
}

1. Department of Health Services Management, North Tehran Branch, Islamic Azad University, Tehran, Iran

2. Health Economics Policy Research Center, Tehran Medical Sciences Branch, Islamic Azad University, Tehran, Iran

3. Department of Health Policy, School of Management and Medical Education, Shahid Beheshti University of Medical Sciences, Tehran, Iran

4. Health Management Research Center, Baqiyatallah University of Medical Sciences, Tehran, Iran creasing due to demographic changes in the population and epidemiological transition; and the demand for these services has increased in developed countries compared to the past (5). Studies have shown that the mortality rate of the patients who need to be admitted to the intensive care unit but not accepted is $24 \%$ more than the admitted patients (6). Intensive care units are costly and important units of hospitals. In 2005, Haplern and Pastores reported that ICU costs account for $15 \%$ of the total costs of the hospitals and $4.1 \%$ of the US health costs; they also found

$\uparrow$ What is "already known” in this topic:

There is no report on designing a similar tool, and this tool was designed for the first time in this study.

\section{$\rightarrow$ What this article adds:}

Designing and validating a measuring tool to identify factors affecting the distribution of hospitals' intensive care beds can be a big step forward in distribution of such beds. 
that the patients' treatment in these wards is 6 times more expensive than other wards in the United States (7). In addition, the importance and application of intensive care beds in rehabilitation is undeniable.

In developing countries, due to the lack of information, skills, and expertise in health and treatment planning, resources are often unevenly allocated (8).

Inequality in the distribution of intensive care beds may increase the problems associated with bed shortage (9).

While many studies have been conducted in developed and developing countries to measure the extent of inequality in the distribution of health care resources, such as human resource and medical equipment, few studies have been done on inequality in distribution of intensive care beds. No study has ever been conducted to develop a tool for measuring the factors affecting the distribution of intensive care beds. Mesgarpour et al, using Gini index, examined fair distribution of intensive care beds. The findings of their study indicated that intensive care beds, especially coronary care unit (CCU) beds, had equal distribution (10). In another study, Omrani Khou et al showed that the distribution of noncardiac intensive care beds and human resources had a fair geographical distribution, but the distribution of NICU beds and kidney transplantation beds was not fair (11). In Maher et al study, a Gini coefficient of 0.281 indicated injustice in distribution of intensive care beds (12).

Hospital beds are the most important criterion for calculating other resources needed, including physicians, nurses and equipment; also, equality in the distribution of intensive care beds can implicitly lead to equality in distribution of other factors of provision of intensive care services (13). Also, the distribution of intensive care beds in the hospitals of Iran has always been challenging and has always had a high occupation coefficient. Thus, the patients have always been forced to wait in long queues, and consequently young people have always been deprived of these services, leading to losing fruitful days and years of life. Therefore, it seems that measuring and examining the factors affecting the distribution of these types of beds can be a big help for equitable distribution and increased access. Thus, this study was conducted to design and validate a measuring tool for the factors affecting the distribution of hospitals' intensive care beds in Iran in 2018.

\section{Methods}

This mix study was conducted from April to October 2018. In the first step, all literature on factors affecting the distribution of intensive care beds in Iran and other countries were reviewed. A total of 800 articles, documents, and books were searched in the first step. Adter examining data entry criteria, such as factors affecting the distribution of hospital beds in discussion and conclusion sections, 35 documents were used for item registration. Finally, 60 items related to distribution of hospital beds were extracted. In the second step, face and content validity of the primary questionnaire was assessed by 20 experts and specialists with several years of experience in management and policymaking of various macro areas of health system of Iran. To determine the formal validity using a qualitative method, the primary questionnaire was examined for understandability, relatedness, and ambiguity by the expert panel. Vague phrases or those not appropriate in wording or content were edited, and after 3 revisions, the list of affecting factors was developed and formulated. Moreover, to determine the formal validity of the questionnaire using a quantitative method, to reduce or eliminate inappropriate phrases, and to determine the importance of each item, item impact score method was used. Finally, a questionnaire was formulated and the items were presented to the experts in the form of a 5point Likert scale ( $1=$ not important; $2=$ has little importance; 3 = has moderate importance; 4 = relatively important; and $5=$ very important). Then, the impact point of each item was calculated according to the following equation: The impact item $=$ item frequency $(\%) *$ item importance. Then, the mean score was calculated by multiplying the scores assigned to each item by the ratio of those who had selected 4 and 5 choices, and items with an average above 1.5 were accepted (14). To confirm content validity, qualitative and quantitative methods were considered and the questionnaire was examined for wording, adaptation of the items with related areas, and understandability of the items in three 2-hour sessions in 3 consequence days. The comments were applied to the questionnaire and the content validity ratio (CVR) and Lawshe approach were used to examine the qualitative validity of the questionnaire (15). The formula of content validity ratio is $C V R=(\mathrm{Ne}-\mathrm{N} / 2) /(\mathrm{N} / 2)$, where the $\mathrm{Ne}$ is the number of panelists indicating "essential" and $\mathrm{N}$ is the total number of panelists. Thus, to ensure the necessity of the questions, the expert panel was asked to comment on each item according to a 3-point Likert scale. For each item, one of the 3 choices of "not necessary", "useful but not necessary", or "necessary" were considered. The numeric value of content validity ratio is determined by Lawshe Table. In this study, in which the number of panelists is 20 , if CVR is bigger than 0.42 , then, the item in the instrument with an acceptable level of significance will be accepted.

To ensure that the items of the measurement tool were developed in the best way possible, the quantitative method of credit index was used to measure the content. Also, to evaluate content validity index, Waltz \& Bausell method was used (16). According to this index, first, the relevancy of the whole phrase was measured. Therefore, the experts were asked to examine the 3 items of "relevancy", "clarity", and "simplicity" for each item of the questionnaire according to a 4-point Likert scale $(1=$ not clear; $2=$ relatively clear; $3=$ clear; and $4=$ very clear $)$. The simplicity feature was rated from 1 to $4(1=$ not simple; $2=$ relatively simple; 3 = simple; and $4=$ very simple). Then, the following formula was used to calculate the content validity index (CVI):

$\mathrm{CVI}=$ the number of experts giving a rating of "very relevant" and "relevant" for each item divided by the total number of experts (17).

In the next step, according to the mean scores of the content validity indices for all items of the questionnaire and the mean score of all the 3 indices, the mean score of 
the content validity index (S-CVI/Ave) was calculated (18). Cronbach's alpha and test-retest were also used to determine the internal consistency of the questionnaire (19).

\section{Results}

The factors affecting the distribution of intensive care beds, with 60 items, were categorized into 6 dimensions: demographic information (6 items), geographic (11 items, economic (10 items), sociopolitical (11 items), organizational (11 items), and structural (11 items) questions. After conducting face validity and finalizing the qualitative stage to determine content validity, items 14, 10, 56, and 18 were merged. Then, through a quantitative content validity examination and calculating CVR, 34 items obtained a CVR score more than 0.42 . The results of CVI showed that all 34 remained items had scores of higher than 0.81 . Content validity ratio (CVR) and content validity index (CVI) was calculated to be 0.88 and 0.89 for the whole questionnaire, respectively.

The results of Table 1 revealed that organizational and constructional components had higher impact.

The value of (S-CVI/Ave) was also obtained to be 0.92 . The values of Cronbach's alpha (0.747) (Table 2) and Spearman correlation (0.81) were also confirmed, and the final questionnaire, with 34 items, was confirmed (Appendix).

\section{Discussion}

In this study, a 34-item questionnaire covering 6 areas (demographic, geographic, economic, sociopolitical, organizational, and structural) were designed to identify the factors affecting the distribution of beds in intensive care wards of hospitals.

Extensive literature review revealed that these factors are important in the distribution of beds, service quality, and equality in access and beds management.

Nowadays, planning, decisionmaking, and resources allocation in health care systems intensely depend on performance evaluation of the system, and for such evaluation, appropriate measuring tools are needed (20). The validation process was done using key expert's opinions.

Population, bed occupation coefficient, and human resource are factors affecting the deployment of intensive care beds. Salamzadeh, in his research, pointed out that human resource is the most important item among the factors affecting efficiency and suggested that increasing the percentage of occupied beds and its performance requires a comprehensive and long-term planning (21).

In the present study, many important variables related to distribution of beds were extracted, such as number of physicians, number of nurses, bed occupation coefficient, average length of stay, and equipment. Colin \& Bell introduced bed occupation coefficient as the most important and practical index in measuring the efficiency of the hospital that indicates the performance of the hospital (22). Also, McClean introduced a model for the number of beds in delivery room, where he identified the bed occupancy rate as bed allocation efficiency. McClean et al believed that this factor alone is not sufficient to measure the efficiency and that a set of other factors, such as the number of physicians and nurses, equipment of the ward, and the average length of stay are also involved (23). Cohen et al, in their study, found that despite a high demand for hospital beds, a large number of these beds are unused due to improper planning and management. Given that the percentage of hospital beds occupation in different wards of a hospital can depend on the performance of management, physicians, and other staff of hospitals, the changes in the percentage of hospital beds occupation can be an effective factor in distribution of hospital beds and services (24).

In the present study, organizational and structural areas had a high impact coefficient. Lignin et al, reported that management of hospital beds includes managing all admissions, duration of stay in the hospital, discharging and inter transition and intra transition in a systematic framework. Moreover, they indicated that a change in the number of beds can change human and financial resources of each ward. Different factors contribute to redistribution of hospital beds, including bed occupation rate, patient waiting list, and the number of human resource (25).

The findings of the present study also showed that hu-

Table 1. The percentage of impact factor for different parts of the questionnaire and factors affecting the distribution of hospitals' intensive care beds in Iran

\begin{tabular}{lcc}
\hline Areas & Total items' coefficient impact & The percentage of impact coefficient \\
\hline Demographic & 16.3 & $14.1 \%$ \\
Geographical & 17.4 & $15.2 \%$ \\
Economic & 13.8 & $12.1 \%$ \\
Sociopolitical & 16.6 & $14.4 \%$ \\
Organizational & 29.4 & $25.6 \%$ \\
Constructional & 21.5 & $18.6 \%$ \\
Total areas & 115 & $100 \%$ \\
\hline
\end{tabular}

Table 2. Reliability of the factors affecting the distribution of hospitals' intensive care beds in Iran

\begin{tabular}{lcc}
\hline Areas & Number \& cod of each item in the area & Cronbach's alpha \\
\hline Demographic & $5(1-5)$ & 0.745 \\
Geographical & $5(6-10)$ & 0.752 \\
Economic & $5(11-15)$ & 0.738 \\
Social-Political & $5(16-20)$ & 0.741 \\
Organizational & $7(21-28)$ & 0.763 \\
Constructional & $7(28-34)$ & 0.747 \\
Total areas & 34 Items & 0.747 \\
\hline
\end{tabular}


man resources, including physicians, nurses, and anesthesiologists, affect the distribution of intensive care beds. Mark et al, in their study, found a direct relationship between the number of nurses and the costs (26). The findings of this research also showed that if the nursing staff is proportional to the number of beds, the side-effects and the duration of patient stay in the hospital will decrease and the care and supervision will improve.

In this study, the average length of stay- maximum distance to access the hospital services and access time- were extracted as variables with high impact coefficients.

Rashidian et al, in their study, showed that the decrease in the average length of infants stay on NICU beds from 20 days to 10 days reduced the need for NICU beds to half. In this study, the access distance is mentioned as the main point of distinction between this study and former studies (27).

In the present study, the population and age distribution of the population were extracted as 2 important factors affecting the distribution of intensive care beds. Agha Mohammadi et al, in their study, stated that decisionmaking about the distribution of hospital beds has different dimensions, one of which being population structure (28).

Also, in the current study, some affecting factors, such as population and the average length of stay in the hospital and common diseases that lead to readmission, were more important and had a higher impact coefficient given that the number of readmission, population, and the average length of stay are 3 factors involved in the calculation of the number of required beds. Comparing the sum of impact coefficients for each area indicated that structural and organization factors had higher scores. The collection of rules and regulations classification and national and localized planning in a ministerial level (4.9 out of 5 points) had the highest impact coefficient among affecting factors.

The high impact for organizational and structural factors can be justified with experience of experts. All participants of the study were experienced managers, experts, and specialists in the health care system of the country (Table 1). However, these factors may be less important in view of service providers and the service receivers. Therefore, considering the opinion of service providers and service receivers is a necessity.

Also, the extracted factors that affect the distribution of intensive care beds from experts' point of view are merely applicable to the intensive care beds of the country and these factors may change when considering other hospital beds and other clinical groups, which was the limitation of this study.

\section{Conclusion}

This study was conducted for the first time in Iran. Also, given that the distribution of intensive care beds in the hospitals of Iran has always been challenging, identifying the most important factors and using a measuring tool is of high importance. Thus, it seems that measuring and examining the factors affecting the distribution of intensive care beds can help achieve equitable distribution of such beds, and an appropriate pattern can be accomplished for redistribution of intensive care beds in Iran.

\section{Conflict of Interests}

The authors declare that they have no competing interests.

\section{References}

1. Silva Junior AJ, Posse MB, de Violoncellos Nebo LC. Hospital bed project for home care. Rev Esc Enferm USP. 2010;44(2):302-7.

2. Panis LJ, Gooskens M, Verheggen. Predictors of inappropriate hospital stay: a clinical study. Int J Qual Health Care. 2013;15(1):57-65.

3. Miao CH, Zhuo L, Uming G. Study of large Medical equipment allocation in Xuzhou. J Zhejiang Uni Science B. 2010;8(12):881-884.

4. Lawes CM, Hoorn SV, Rodgers A. Global burden of blood-pressurerelated disease, 2011. The Lancet. 2009;371(9623):1513-8.

5. Wunsch H, Angus DC, Harrison DA, Collange O, Fowler R, Hoste AJ, et al. Variation in critical care services across north America and western Europe. Critic Care Med. 2009;26(10):2087-93.

6. McIntyre D, Mooney G, editors. The economics of health equity. Cambridge University Press; 2007 Oct 11.

7. Halpern NA, Pastores SM. Critical care medicine in the United States 2000-2005: an analysis of bed numbers, occupancy rates, payer mix, and costs. Critic Care Med. 2010 Jan 1;38(1):65-71.

8. Dejian L, Jin H, Jan M, Risser Asha S. Statistical Properties of Generalized Gini Coefficient with Application to Health Inequality Measurement. Springer Sci. 2009; 687:209-238.

9. Tofighi S, Meskarpour Amiri M, Ameriuon A, Naseri H. Equity in distribution of intensive care beds in Iran with Gini coefficient and Lorenz curve approach. Yafteh. 2011;12(2):75-83.

10. Meskarpour-Amiri M, Mehdizadeh P, Barouni M, Dopeykar $\mathrm{N}$, Ramezanian M. Assessment the trend of inequality in the distribution of intensive care beds in Iran: using GINI index. Glob J Health Sci. 2014,25;6(6):28-36.

11. Omrani-khoo H,Lotf F ,Safari H, Zargar S, Jame B, Moghri J, et al, Equity in Distribution of Health Care Resources; Assessment of Need and Access, Using Three Practical Indicators. Iran J Pub Health. 2013;42(11):1299-1308.

12. Maher A, Marzang S, Hosseini SM. Correlation between Distribution of Hospital Inpatient Beds and Households expenditure in Iran. J Health Based Res. 2016;2(3):275-287. (Persian)

13. de Bruin AM, Bekker R, Van Zanten L, Koole GM. Dimensioning hospital wards using the Erlang loss model. Ann Operat Res. 2010 Jul 1;178(1):23-43.

14. Yaghmaie F. Content validity and its estimation. J Med Educ. 2003;3(1):25-7. (Persian)

15. Lawshe $\mathrm{CH}$. A quantitative approach tocontentvalidity. Person Psychol. 1975;28(4):563-75.

16. Waltz C, Strickland O, Lenz E. Measurement in Nursing and Health Research. Springer Publishing Company. New York, NY. 2010.

17. Hyrkas K, Schmidlechner KA, Oksa L. Validating on Instrument for Clinical Supervision using an Expert Panel. Int $\mathrm{J}$ Nurs Stud. 2013;20(6):219-125.

18. Polit DF. Beck CT, Owen SV. Is the CVI an Acceptable Indicator of Content Validity, Appraisal and Recommendations. Res Nurs Health. 2009;36:419-367.

19. Bland JM, Atman DG. Statistic Note: Cronbach Alpha. BMJ (Clinical research ed.) 1997;7080:314-572.

20. Scott IA. Public hospital bed crisis: too few or too misused? Aust Health Rev. 2010;34(3):317-24.

21. Salam Zadeh Y, Mansoori H, Farid D. Determining nursing manpower needed in a sample hospital. Hospital. 2011;9(3-4):69-76. (Persian)

22. Calman KC. The ethics of allocation of scarce health care resources: a view from the center. J Med Ethics. 1994;20(2):71-74.

23. McClean J. "Income related inequity in health care access and delivery", PHD, University of South Carolina. 2014.

24. Cohen Martin and Myfanuy. Access to health care, New'Ftter Lan, London. 2013.

25. Lignin T, Pesis-Katz I, Mukamel DB. "Trends in geographic disparities in allocation of health care resources in the US." Health Policy. 2014;68(2):223-232.

26. Bay MS, Nestman LJ. The Use of Bed Distribution and Service population Indexes for Hospital Bed Allocation. Health Serv Res. 1984 
Jun; 19(2):141-160

27. Rashidian A. The comprehensive health coveragein Iran. Health J. 2010. (Persian)

28. Aghamohamadi S, Khosravi A, Safari R. Improving the Quality and Use of Birth, Death and Causeof-Death Information" Guidance for a standardsbased review of country practices" [Persian] Iranian Ministry of Health and Medical Education Deputy for Health. 2016:14-40. (Persian) 